\title{
Dynamical Analysis and Cosmological Evolution in Weyl Integrable Gravity
}

\author{
Andronikos Paliathanasis 1,2 (D) \\ 1 Institute of Systems Science, Durban University of Technology, P.O. Box 1334, Durban 4000, South Africa; \\ anpaliat@phys.uoa.gr \\ 2 Instituto de Ciencias Físicas y Matemáticas, Universidad Austral de Chile, Valdivia 5090000, Chile
}

Citation: Paliathanasis, A.

Dynamical Analysis and

Cosmological Evolution in Weyl Integrable Gravity. Universe 2021, 7,

468. https://doi.org/10.3390/

universe7120468

Academic Editor: Lorenzo Iorio

Received: 27 October 2021

Accepted: 29 November 2021

Published: 30 November 2021

Publisher's Note: MDPI stays neutral with regard to jurisdictional claims in published maps and institutional affiliations.

Copyright: (C) 2021 by the author. Licensee MDPI, Basel, Switzerland. This article is an open access article distributed under the terms and conditions of the Creative Commons Attribution (CC BY) license (https:/ / creativecommons.org/licenses/by/ $4.0 /)$.

\begin{abstract}
We investigate the cosmological evolution for the physical parameters in Weyl integrable gravity in a Friedmann-Lemaître-Robertson-Walker universe with zero spatial curvature. For the matter component, we assume that it is an ideal gas, and of the Chaplygin gas, from the Weyl integrable gravity a scalar field is introduced by a geometric approach which provides an interaction with the matter component.We calculate the stationary points for the field equations and we study their stability properties. Furthermore, we solve the inverse problem for the case of an ideal gas and prove that the gravitational field equations can follow from the variation of a Lagrangian function. Finally, variational symmetries are applied for the construction of analytic and exact solutions.
\end{abstract}

Keywords: cosmological dynamics; Weyl integrable theory; scalar field; interaction

\section{Introduction}

The cosmological constant component in the Einstein-Hilbert Action Integral is the simplest dark energy candidate to describe of the recent acceleration phase of the universe, as it is provided by the cosmological observations [1]. In the so-called $\Lambda C D M$ cosmology the universe is considered to be homogeneous and isotropic, described by the FriedmannLemaître-Robertson-Walker (FLRW) geometry with spatially flat term, where the matter component consists of the cosmological constant and a pressureless fluid source which attributes the dark matter component of the universe. The gravitational field equations are of second-order and can be integrated explicitly. Indeed, the field equations can be reduced to that of the one-dimensional "hyperbolic oscillator". However, as the cosmological observations are improved, $\Lambda$-cosmology loses the important position in the "armoury" of cosmologists. For an interesting discussion on the subject we refer the reader to the recent review [2]. Furthermore, because of the simplicity of the field equations in $\Lambda$-cosmology, the cosmological constant term cannot provide a solution for the description of the complete cosmological evolution and history.

In order to solve these problems, cosmologists have introduced various solutions in the literature by introducing new degrees of freedom in the field equations. Time-varying $\Lambda$ term, scalar fields and fluids with time-varying equation of state parameters, like the Chaplygin gases have been proposed to modify the energy-momentum tensor of the field equations [3-8]. On the other hand, a different approach is inspired by the modification of the Einstein-Hilbert Action integral, and leads to the family of theories known as alternative/modified theories of gravity [9-11]. Another interesting consideration is the interaction between the various components of the energy momentum tensor [12]. Interaction in the dark components of the cosmological model, that is, between, the dark energy and the dark matter terms, is supported by cosmological observations [13-16].

For a given proposed dark energy mode model, there are systematic methods for the investigation of the physical properties of the model. The derivation of exact and analytic solutions is an essential approach because analytic techniques can be used for the investigation of the cosmological viability of the model [17-20]. Furthermore, from the 
analysis of the asymptotic dynamics, that is, of the determination of the stationary points, the complete cosmological history can be constructed [21-23]. Indeed, constraints for the free parameters of a given model can be constructed through the analysis of the stationary points and the specific requirements for the stability of the stationary points [24-28].

In this piece of work, we study the evolution of the cosmological dynamics for the theory known as Weyl integrable gravity (WIG) [29-35]. In WIG a scalar field is introduced into the Einstein-Hilbert Action Integral by a geometric construction approach. Indeed, in Riemannian geometry the basic geometric object is the covariant derivative $\nabla_{\mu}$ and the metric tensor $g_{\mu \nu}$, such that it has no metricity component, i.e., $\nabla_{\kappa} g_{\mu v}=0$ [36]. In Weyl geometry the fundamental geometric objects are the gauge vector field $\omega_{\mu}$ and the the metric tensor $g_{\mu v}$, such that $\tilde{\nabla}_{\kappa} g_{\mu v}=\omega_{\kappa} g_{\mu \nu}$, where now $\tilde{\nabla}_{\mu}$ notes the covariant derivative with respect to the affine connection $\tilde{\Gamma}_{\mu \nu}^{\kappa}$ which is defined as $\tilde{\Gamma}_{\mu \nu}^{\kappa}=\Gamma_{\mu \nu}^{\kappa}-$ $\omega_{(\mu} \delta_{v)}^{\kappa}+\frac{1}{2} \omega^{\kappa} g_{\mu \nu}$. When $\omega_{\mu}$ is defined by a scalar field $\phi, \tilde{\Gamma}_{\mu \nu}^{\kappa}$ describes the affine connection for the conformal metric $\tilde{g}_{\mu \nu}=\phi g_{\mu v}$. The field equations of the WIG in the vacuum are equivalent to that of General Relativity with a massless scalar field, with positive or negative energy density. However, when a matter source is introduced, interaction terms appear as a natural consequence of the geometry of the theory [36]. In geometric terms of the interaction context, we investigate the dynamics of the cosmological field equations so that we construct the cosmological history and investigate the viability of the theory. Furthermore, the integrability property for the field equations is investigated by using the method of variational symmetries for the determination of conservation laws.

In Section 2 we present the basic elements for the WIG theory. Furthermore, we write the field equations for our cosmological model in a spatially flat FLRW background space. In Section 3 we present the main results of our analysis in which we discuss the asymptotic dynamics for the field equations in the cases for which the matter source is an ideal gas, or a Chaplygin gas. Moreover, we investigate the dynamics in the presence of the cosmological constant term. In Section 4 we show that the field equations have a minisuperspace description when the matter source is an ideal gas. Specifically, we solve the inverse problem and we construct a point-like Lagrangian which describes the cosmological field equations. With the use of the variational symmetries we determine a conservation law and we present the analytic solution for the field equations by using the Hamilton-Jacobi approach. Our results are summarized in Section 5.

\section{Weyl Integrable Gravity}

Consider the two conformal related metric tensors $g_{\mu v}, \tilde{g}_{\mu v}$ such that $\tilde{g}_{\mu v}=\phi g_{\mu v}$. The Christoffel symbols of the two conformal related metrics are related as

$$
\tilde{\Gamma}_{\mu \nu}^{\kappa}=\Gamma_{\mu \nu}^{\kappa}-\phi_{,(\mu} \delta_{v)}^{\kappa}+\frac{1}{2} \phi^{, \kappa} g_{\mu \nu} .
$$

In Weyl geometry the fundamental objects are the metric tensor $g_{\mu \nu}$ and the covariant derivative $\tilde{\nabla}_{\mu}$ defined by the Christoffel symbols $\tilde{\Gamma}_{\mu \nu}^{\kappa}$. Hence, the curvature tensor is defined

$$
\tilde{\nabla}_{v}\left(\tilde{\nabla}_{\mu} u_{\kappa}\right)-\tilde{\nabla}_{\mu}\left(\tilde{\nabla}_{v} u_{\kappa}\right)=\tilde{R}_{\kappa \lambda \mu v} u^{\lambda} .
$$

Consequently, the Ricci tensors of the two conformal metrics are related as follows:

$$
\tilde{R}_{\mu v}=R_{\mu \nu}-\tilde{\nabla}_{v}\left(\tilde{\nabla}_{\mu} \phi\right)-\frac{1}{2}\left(\tilde{\nabla}_{\mu} \phi\right)\left(\tilde{\nabla}_{\nu} \phi\right)-\frac{1}{2} g_{\mu \nu}\left(\frac{1}{\sqrt{-g}} \tilde{\nabla}_{v} \tilde{\nabla}_{\mu}\left(g^{\mu \nu} \sqrt{-g} \phi\right)-g^{\mu \nu}\left(\tilde{\nabla}_{\mu} \phi\right)\left(\tilde{\nabla}_{\nu} \phi\right)\right),
$$

thus the Ricci scalar

$$
\tilde{R}=R-\frac{3}{\sqrt{-g}} \tilde{\nabla}_{\nu} \tilde{\nabla}_{\mu}\left(g^{\mu \nu} \sqrt{-g} \phi\right)+\frac{3}{2}\left(\tilde{\nabla}_{\mu} \phi\right)\left(\tilde{\nabla}_{\nu} \phi\right) .
$$


In WIG the the fundamental Action Integral is defined by using the Weyl Ricci scalar $\tilde{R}$ and the scalar field $\phi$ by the expression

$$
S_{W}=\int d x^{4} \sqrt{-g}\left(\tilde{R}+\xi\left(\tilde{\nabla}_{\nu}\left(\tilde{\nabla}_{\mu} \phi\right)\right) g^{\mu \nu}-\Lambda\right),
$$

where $\xi$ is a coupling constant. From (5) we observe that $\phi$ is a massless scalar field. However, more generally, a potential function may be considered.

From the Action Integral (5) the Weyl-Einstein equations are as [36]

$$
\tilde{G}_{\mu \nu}+\tilde{\nabla}_{\nu}\left(\tilde{\nabla}_{\mu} \phi\right)-(2 \xi-1)\left(\tilde{\nabla}_{\mu} \phi\right)\left(\tilde{\nabla}_{\nu} \phi\right)+\xi g_{\mu \nu} g^{\kappa \lambda}\left(\tilde{\nabla}_{\kappa} \phi\right)\left(\tilde{\nabla}_{\lambda} \phi\right)-\Lambda g_{\mu \nu}=0,
$$

where $\tilde{G}_{\mu \nu}$ is the Weyl Einstein tensor. By using the Riemannian Einstein tensor $G_{\mu \nu}$, the Weyl-Einstein field Equations (6) become [36]

$$
G_{\mu \nu}-\lambda\left(\phi, \mu \phi_{, v}-\frac{1}{2} g_{\mu \nu} \phi^{, \kappa} \phi, \kappa\right)-\Lambda g_{\mu \nu}=0,
$$

where $\lambda$ is defined as $2 \lambda \equiv 4 \xi-3$. Equations (7) are nothing else than the field equations of Einstein's General Relativity with a massless scalar field. When $\lambda>0$, the scalar field $\phi$ is a quintessence while, when $\lambda<0, \phi$ is a phantom field [36] is [36]

Moreover, for the equation of motion of the scalar field $\phi$, the Klein-Gordon equation

$$
\left(\tilde{\nabla}_{\nu}\left(\tilde{\nabla}_{\mu} \phi\right)\right) g^{\mu \nu}+2 g^{\mu \nu}\left(\tilde{\nabla}_{\mu} \phi\right)\left(\tilde{\nabla}_{\nu} \phi\right)=0,
$$

or by using the Riemannian covariant derivative $\nabla_{\mu}$, expression (8) is written in the usual form $g^{\mu \nu} \nabla_{\nu} \nabla_{\mu} \phi=0$.

As it was found in [36], the introduction of a perfect fluid in the gravitational model leads to the following set of gravitational field equations [36]

$$
\tilde{G}_{\mu \nu}+\tilde{\nabla}_{\nu}\left(\tilde{\nabla}_{\mu} \phi\right)-(2 \xi-1)\left(\tilde{\nabla}_{\mu} \phi\right)\left(\tilde{\nabla}_{\nu} \phi\right)+\xi g_{\mu v} g^{\kappa \lambda}\left(\tilde{\nabla}_{\kappa} \phi\right)\left(\tilde{\nabla}_{\lambda} \phi\right)-\Lambda g_{\mu \nu}=e^{-\frac{\phi}{2}} T_{\mu v}^{(m)}
$$

that is,

$$
G_{\mu \nu}-\lambda\left(\phi, \mu \phi, v-\frac{1}{2} g_{\mu \nu} \phi^{, \kappa} \phi_{, \kappa}\right)-\Lambda g_{\mu \nu}=e^{-\frac{\phi}{2}} T_{\mu \nu}^{(m)},
$$

where $T_{\mu \nu}^{(m)}=\left(\rho_{m}+p_{m}\right) u_{\mu} u_{v}+p_{m} g_{\mu \nu}$.

Moreover, the modified Klein-Gordon equation follows [36]

$$
-g^{\mu \nu} \nabla_{\nu} \nabla_{\mu} \phi=\frac{1}{2 \lambda} e^{-\frac{\phi}{2}} \rho_{m}
$$

Equation (11) follows from the identity $G_{; v}^{\mu v}=0$, which provides the conserve of the effective energy-momentum tensor.

\section{FLRW Spacetime}

Following the cosmological principle, in very large scales the universe is considered to be isotropic and homogeneous. Hence, the physical space is described by the FLRW spacetime, where the three-dimensional surface is a maximally symmetric space and admits six isometries. However, from cosmological observations the spatial curvature is very small, which means that we can consider as background space the spatially flat FLRW metric

$$
d s^{2}=-d t^{2}+a^{2}(t)\left(d r^{2}+r^{2}\left(d \theta^{2}+\sin ^{2} \theta d \varphi^{2}\right)\right) .
$$


Moreover, we assume the co-moving observer $u_{\mu}=\delta_{\mu}^{t}$, with expansion rate $\theta=3 \frac{\dot{a}}{a}$, for the line element (12) and for a scalar field $\phi=\phi(t)$, the gravitational field equations are

$$
\begin{gathered}
\frac{\theta^{2}}{3}-\frac{\lambda}{2} \dot{\phi}^{2}-\Lambda-e^{-\frac{\phi}{2}} \rho_{m}=0, \\
\dot{\theta}+\frac{1}{3} \theta^{2}+\frac{1}{2} e^{-\frac{\phi}{2}}\left(\rho_{m}+3 p_{m}\right)+\lambda \dot{\phi}^{2}-\Lambda=0, \\
\ddot{\phi}+\theta \dot{\phi}+\frac{1}{2 \lambda} e^{-\frac{\phi}{2}} \rho_{m}=0
\end{gathered}
$$

and

$$
\dot{\rho}_{m}+\theta\left(\rho_{m}+p_{m}\right)-\rho_{m} \dot{\phi}=0 .
$$

From the modified Friedmann equations we observe the existence of a non-zero interacting term for scalar field $\phi$ and the matter component $\rho_{m}$. When $\lambda>0$, energy decays from scalar field to the $\rho_{m}$, while for $\lambda<0$ energy decays from $\rho_{m}$ to the field $\phi$. Furthermore, the effective equation of state parameter for the effective cosmological matter is defined as $w_{\text {eff }}=-1-2 \frac{\dot{\theta}}{\theta^{2}}$.

Finally, for the nature of the matter source $\rho_{m}$ in the following we consider that $\rho_{m}$ is an ideal gas, or a Chaplygin gas.

\section{Cosmological Dynamics}

We continue our analysis with the investigation of the stationary points for the cosmological field equations. In order to proceed with the study we define the new dimensionless variables in the context of $\theta$-normalization

$$
x=\sqrt{\frac{3}{2}} \frac{\dot{\phi}}{\theta}, \Omega_{\Lambda}=\frac{3 \Lambda}{\theta^{2}}, \Omega_{m}=\frac{3 \rho_{m}}{\theta^{2}} e^{-\frac{\phi}{2}}
$$

where for the equation of state parameter for the matter source we consider (i) ideal gas $p_{m}=(\gamma-1) \rho_{m}, 0 \leq \gamma<2$, and (ii) Chaplygin gas $p_{m}=\frac{A_{0}}{\rho_{m}^{\alpha}}, \alpha \geq 1$. Moreover, we define the new independent parameter to be $\tau=\ln (a)$, such that $x^{\prime}=\frac{d x}{d \tau}$.

At the stationary points the effective equation of the state parameter is defined as $w_{\text {eff }}=w_{\text {eff }}\left(x, \Omega_{\Lambda}, \Omega_{m}\right)$, so that the asymptotic solution is described by the scale factor $a(t)=a_{0} t^{\frac{2}{3\left(1+w_{e f f}\right)}}, w_{e f f} \neq-1$ and $a(t)=a_{0} e^{H_{0} t}$, when $w_{\text {eff }}=-1$.

\subsection{Ideal Gas with $\Lambda=0$}

Assume the equation of state of an ideal gas $p_{m}=(\gamma-1) \rho_{m}$, without the cosmological constant term. Then in the new dimensionless variables (17) the field equations are

$$
\begin{gathered}
\Omega_{m}=1-\lambda x^{2}, \\
x^{\prime}=-\frac{\left(1-\lambda x^{2}\right)(\sqrt{6}-6(\gamma-2) \lambda x)}{12 \lambda} .
\end{gathered}
$$

Moreover, $\Omega_{m}$ is bounded as $0 \leq \Omega_{m} \leq 1$, such that the solution is physically acceptable, that is, from (18) it follows that there are physical stationary points only when $\lambda>0$.

The stationary points of Equation (19) are

$$
A_{1}^{ \pm}: x_{1}^{ \pm}=\frac{1}{\sqrt{\lambda}}, A_{2}: x_{2}=\frac{1}{\sqrt{6}(\gamma-2) \lambda} .
$$

Points $x_{1}^{ \pm}$describe asymptotic solutions where only the scalar field contributes to the cosmological fluid. The effective equation of state parameter is derived to be $w_{\text {eff }}\left(x_{1}^{ \pm}\right)=1$, from which we infer that the solution is that of a stiff fluid. On the other hand, the point $x_{2}$ 
is physically acceptable when $\lambda \geq \frac{1}{6(\gamma-2)^{2}}$, and the point describes a scaling solution with $w_{\text {eff }}\left(x_{2}\right)=-1+\gamma+\frac{1}{6 \lambda(\gamma-2)}$. For $\gamma<\frac{2}{3}, \lambda>\frac{1}{8(1-2 \gamma)+6 \gamma^{2}}$ it follows that $w_{\text {eff }}\left(x_{2}\right)<-\frac{1}{3}$ which means that the asymptotic solution describes an accelerated universe, where in the limit $\lambda=\frac{1}{8(1-2 \gamma)+6 \gamma^{2}}$, the asymptotic solution is that of the de Sitter universe.

We proceed with the investigation of the stability properties for the stationary points. We linearize Equation (19) and we find the eigenvalues $e_{1}\left(x_{1}^{ \pm}\right)=2-\gamma \mp \frac{1}{\sqrt{6 \lambda}}, e_{1}\left(x_{2}\right)=$ $-1+\frac{\gamma}{2}+\frac{1}{12 \lambda(2-\gamma)}$. Thus, point $x_{1}^{-}$is always a source, $x_{1}^{+}$is an attractor when $\lambda<\frac{1}{6(\gamma-2)^{2}}$, while $x_{2}$ is the unique attractor when it exists.

\subsection{Ideal Gas with $\Lambda \neq 0$}

In the presence of the cosmological constant, that is, $\Lambda \neq 0$, and when the matter term is that of the ideal gas, the field equations are written as follows

$$
\begin{gathered}
\Omega_{m}=1-\lambda x^{2}-\Omega_{\Lambda} \\
\Omega_{\Lambda}^{\prime}=-\Omega_{\Lambda}\left((\gamma-2) \lambda x^{2}+\gamma\left(\Omega_{\Lambda}-1\right)\right)
\end{gathered}
$$

and

$$
x^{\prime}=\frac{1}{12 \lambda}\left(\left(\lambda x^{2}-1\right)(\sqrt{6}-6(\gamma-2) \lambda x)+(\sqrt{6}-6 \gamma \lambda x) \Omega_{\Lambda}\right) .
$$

Furthermore, we assume that $\left|\Omega_{\Lambda}\right| \leq 1$, from which we infer that $x$ is also bounded, and we do not have to study the dynamical system for the existence of stationary points at infinity.

The stationary points of the dynamics system (22), (23) are defined in the plane $\left\{x, \Omega_{\Lambda}\right\}$, that is $B=\left(x(B), \Omega_{\Lambda}(B)\right)$. The points are

$$
\begin{gathered}
B_{1}^{ \pm}=\left( \pm \frac{1}{\sqrt{\lambda}}, 0\right), B_{2}=\left(\frac{1}{\sqrt{6}(\gamma-2) \lambda}, 0\right), \\
B_{3}=(0,1), B_{4}=(\sqrt{6} \gamma, 1+6(2-\gamma) \gamma \lambda) .
\end{gathered}
$$

Points $B_{1}^{ \pm}, B_{2}$ are actually the stationary points $A_{1}^{ \pm}$and $A_{2}$, respectively, for which the cosmological constant component is zero. The physical properties are the same as before. However, we should investigate the stability analysis.

For point $B_{3}$ we derive $w_{\text {eff }}\left(B_{3}\right)=-1, \Omega_{m}\left(B_{3}\right)=0$. Thus point $B_{3}$ describes a de Sitter universe.

Furthermore, point $B_{4}$ provides $\Omega_{m}\left(B_{4}\right)=-12 \gamma \lambda$, $w_{e f f}\left(B_{4}\right)=-1$. The point is physically acceptable when $-\frac{1}{24} \leq \lambda<0$, or $\lambda<-\frac{1}{24}$ with $\gamma \leq-\frac{1}{12 \lambda}$ or $\gamma=0$. The stationary point describes the de Sitter universe in which all the fluid components contribute in the cosmological solution.

We linearize the dynamical system (22), (23) around the stationary points and we derive the eigenvalues. For points $B_{1}^{ \pm}$the eigenvalues are $e_{1}\left(B_{1}^{ \pm}\right)=2-\gamma \mp \frac{1}{\sqrt{6 \lambda}}, e_{2}\left(B_{1}^{ \pm}\right)=2$ from which we infer that $B_{1}^{-}$is always a source, while $B_{1}^{+}$is a saddle point when $\lambda<\frac{1}{6(\gamma-2)^{2}}$. Otherwise it is a source.

For point $B_{2}$ the two eigenvalues are $e_{1}\left(B_{2}\right)=-1+\frac{\gamma}{2}+\frac{1}{12 \lambda(2-\gamma)}, e_{2}\left(B_{2}\right)=\gamma+$ $\frac{1}{6 \lambda(2-\gamma)}$. Thus, point is always a saddle point when it is physically acceptable because $e_{1}\left(B_{2}\right)$ is always negative while $e_{2}\left(B_{2}\right)$ is always positive.

The eigenvalues of the linearized system around the de Sitter point $B_{3}$ are calculated to be $e_{1}\left(B_{3}\right)=-1$ and $e_{2}\left(B_{3}\right)=-\gamma$, from which we infer that the point is always an attractor. Finally, for point $B_{4}$ we find the eigenvalues $e^{ \pm}\left(B_{4}\right)=-\frac{1}{2} \pm \sqrt{1+4 \gamma(1+6(2-\gamma) \lambda)}$. Consequently, point $B_{4}$ is always a saddle point. 


\subsection{Chaplygin Gas with $\Lambda=0$}

Consider now that the matter source satisfies the equation of the state parameter of a Chaplygin gas, $p_{m}=\frac{A_{0}}{\rho_{m}^{\alpha}}$, for which $\alpha \geq 1, A_{0}=(-1)^{\alpha} 3^{-(1+\alpha)} A$ and $\rho_{m} \neq 0$. The field equations are written as follows

$$
x^{\prime}=\frac{1}{12}\left(\frac{(\sqrt{6}+6 \lambda x)\left(\lambda x^{2}-1\right)}{\lambda}+6 x\left(\lambda x^{2}-1\right)^{-\alpha} \gamma\right)
$$

and

$$
Y^{\prime}=\frac{1+\alpha}{6} Y\left(6-\sqrt{6} x+6 \lambda x^{2}+6\left(\lambda x^{2}-1\right)^{-\alpha} Y\right)
$$

where the new variable $Y$ is defined as $Y=A e^{-\frac{1}{2}(1+\alpha) \phi} \theta^{-(2+\alpha)}$.

The stationary points $C=\left(x(C), Y(C)\right.$ ) of the dynamical system (27), (28), with $\Omega_{m}>$

$$
\begin{aligned}
& C_{1}=\left(-\frac{1}{\sqrt{6} \lambda}, 0\right), \\
& C_{2}=\left(\sqrt{\frac{3}{2}}-\frac{\sqrt{\lambda(1+3 \lambda)}}{\sqrt{2} \lambda}, \frac{(\sqrt{3 \lambda(1+3 \lambda)}+6 \lambda(1+3 \lambda-\sqrt{3 \lambda(1+3 \lambda)}))\left(-\frac{1}{2}-3 \lambda \sqrt{3 \lambda(1+3 \lambda)}\right)^{\alpha}}{6 \lambda}\right) \\
& C_{3}=\left(\sqrt{\frac{3}{2}}+\frac{\sqrt{\lambda(1+3 \lambda)}}{\sqrt{2} \lambda}, \frac{(\sqrt{3 \lambda(1+3 \lambda)}+6 \lambda(1+3 \lambda+\sqrt{3 \lambda(1+3 \lambda)}))\left(-\frac{1}{2}+3 \lambda \sqrt{3 \lambda(1+3 \lambda)}\right)^{\alpha}}{6 \lambda}\right) .
\end{aligned}
$$

For point $C_{1}$ we derive $\Omega\left(C_{1}\right)=1-\frac{6}{\lambda}, w_{\text {eff }}\left(C_{1}\right)=\frac{1}{6 \lambda}$. The point is physically acceptable when $\lambda \geq \frac{1}{6}$ while it always describes a universe without acceleration. For $\lambda=\frac{1}{6}$, the asymptotic solution is that of dust, while for $\lambda=\frac{1}{2}$ the asymptotic solution is that of radiation. The eigenvalues of the linearized system around the stationary point are calculated $e_{1}\left(C_{1}\right)=\frac{(1+\alpha)(1+3 \lambda)}{3 \lambda}, e_{2}\left(C_{1}\right)=\frac{1-6 \lambda}{12 \lambda}$, from which we can easily conclude that the stationary point is always a saddle point.

Point $C_{2}$ describes a universe for which $\Omega_{m}\left(C_{2}\right)=\frac{1}{2}-3 \lambda+\sqrt{3 \lambda(1+3 \lambda)}$ and $w_{\text {eff }}\left(C_{2}\right)=\lambda\left(x\left(C_{2}\right)\right)^{2}+\left(\lambda\left(x\left(C_{2}\right)\right)^{2}-1\right)^{-\alpha} Y\left(C_{2}\right)$. The point is well defined when $\lambda>0$, while for large values of $\lambda$ it follows that $w_{\text {eff }}\left(C_{2} ; \lambda>>1\right) \simeq-1$, which means that point $C_{2}$ can describe a solution near to the de Sitter point. On the other hand, point $C_{3}$ is physical acceptable for $0<\lambda \leq \frac{1}{24}$, while we derive $\Omega_{m}=-3 \lambda+\sqrt{3 \lambda(1+3 \lambda)}$ and $w_{\text {eff }}\left(C_{3}\right)=\lambda\left(x\left(C_{3}\right)\right)^{2}+\left(\lambda\left(x\left(C_{3}\right)\right)^{2}-1\right)^{-\alpha} Y\left(C_{3}\right)$ in which $w_{\text {eff }}\left(C_{3} ; \lambda=\frac{1}{24}\right)=1$. Thus point $C_{3}$ does not describe any acceleration.

The eigenvalues of the linearized system near to the stationary points $C_{2}$ and $C_{3}$ are determined. Numerically we find that $e_{1}\left(C_{2}\right), e_{2}\left(C_{2}\right)$ have always negative real parts for $\lambda>0$ and $\alpha \geq 1$; on the other hand $\operatorname{Re}\left(e_{1}\left(C_{3}\right)\right)>0, \operatorname{Re}\left(e_{2}\left(C_{3}\right)\right)>0$ for $\alpha \geq 1,0<\lambda \leq \frac{1}{24}$. Hence, point $C_{2}$ is always an attractor while point $C_{3}$ is always a source.

\subsection{Chaplygin Gas with $\Lambda \neq 0$}

In the presence of a non-zero cosmological constant term, the field equations are reduced to the following dynamical system

$$
\begin{gathered}
\Omega_{m}=1-\lambda x^{2}-\Omega_{\Lambda} \\
\Omega_{\Lambda}^{\prime}=\Omega_{\Lambda}\left(1+\lambda x^{2}-\Omega_{\Lambda}+Y\left(\lambda x^{2}+\Omega_{\Lambda}-1\right)^{-\alpha}\right)
\end{gathered}
$$




$$
\begin{gathered}
x^{\prime}=\frac{1}{12}\left(x^{2}(\sqrt{6}+6 \lambda)+\frac{\sqrt{6}}{\lambda}\left(\Omega_{\Lambda}-1\right)+6 x\left(Y\left(\lambda x^{2}+\Omega_{\Lambda}-1\right)^{-\alpha}-1-\Omega_{\Lambda}\right)\right) \\
Y^{\prime}=\frac{1+\alpha}{6} Y\left(6\left(1+\lambda x^{2}+\Omega_{\Lambda}+Y\left(\lambda x^{2}+\Omega_{\Lambda}-1\right)^{-\alpha}\right)-\sqrt{6} \lambda x\right) .
\end{gathered}
$$

The physically acceptable stationary points $D=\left(x(D), Y(D), \Omega_{\Lambda}(D)\right)$ are

$$
\begin{gathered}
D_{1}=\left(x\left(C_{1}\right), Y\left(C_{1}\right), 0\right), D_{2}=\left(x\left(C_{2}\right), Y\left(C_{2}\right), 0\right), \\
D_{3}=\left(x\left(C_{3}\right), Y\left(C_{3}\right), 0\right), D_{4}=(\sqrt{6}, 1+6 \lambda, 0),
\end{gathered}
$$

where $D_{1}, D_{2}$ and $D_{3}$ have the same physical properties as points $C_{1}, C_{2}$ and $C_{3}$, respectively.

For the point $D_{4}$ we find $\Omega_{m}\left(D_{4}\right)=-12 \lambda$ and $w_{e f f}\left(D_{4}\right)=-1$, which means that the asymptotic solution is physically acceptable when $-\frac{1}{12} \leq \lambda<0$, while the asymptotic solution is that of the de Sitter universe.

The eigenvalues of the linearized system near $D_{1}$ are $e_{1}\left(D_{1}\right)=\frac{(1+\alpha)(1+3 \lambda)}{3 \lambda}, e_{2}\left(D_{1}\right)=$ $\frac{1-6 \lambda}{12 \lambda}$ and $e_{3}\left(D_{1}\right)=\frac{1+6 \lambda}{6 \lambda}$, which means that point $D_{1}$ is always a saddle point. For the points $D_{2}$ and $D_{3}$ we find numerically that $D_{2}$ is always an attractor while $D_{3}$ is always a source. Finally, for the point $D_{4}$ we calculate $e_{1}\left(D_{4}\right)=-(1+\alpha), e_{2}^{ \pm}=\frac{1}{2}(-1 \pm \sqrt{5+24 \lambda})$, from which it follows that the stationary point is always a saddle point.

\section{Minisuperspace Description and Conservation Laws}

For an ideal gas $p_{m}=(\gamma-1) \rho_{m}$, from Equation (16) it follows $\rho_{m}(t)=\rho_{m 0} a^{-3 \gamma} e^{\phi}$ in which $\rho_{m 0}$ is a constant of integration.

We substitute this into the rest of the field equations and we end with the following dynamical system

$$
\begin{gathered}
\frac{\theta^{2}}{3}-\frac{\lambda}{2} \dot{\phi}^{2}-\Lambda-\rho_{m 0} e^{\frac{\phi}{2}} a^{-3 \gamma}=0, \\
\dot{\theta}+\frac{1}{3} \theta^{2}+\frac{(3 \gamma-2)}{2} \rho_{m 0} e^{\frac{\phi}{2}} a^{-3 \gamma}+\lambda \dot{\phi}^{2}-\Lambda=0, \\
\ddot{\phi}+\theta \dot{\phi}+\frac{\rho_{m 0}}{2 \lambda} e^{\frac{\phi}{2}} a^{-3 \gamma}=0 .
\end{gathered}
$$

For the second-order differential Equations (39) and (40) in the space of variables $\{a, \phi\}$, the inverse problem for the determination of a Lagrangian function, provides that the function

$$
L(a, \dot{a}, \phi, \dot{\phi})=-3 a \dot{a}^{2}+\frac{\lambda}{2} a^{3} \dot{\phi}^{2}-a^{3} \Lambda-\rho_{m 0} e^{\frac{\phi}{2}} a^{3-3 \gamma}
$$

is an autonomous Lagrangian function for the field equations, while Equation (38) is conservation law of "energy", i.e., the Hamiltonian $\mathcal{H}$, constraint $\mathcal{H}=0$.

In general, the field equations for the cosmological model in WIG theory with an ideal gas, for the metric

$$
d s^{2}=-N^{2}(t)+a^{2}(t)\left(d x^{2}+d y^{2}+d z^{2}\right),
$$

follow from the singular point-like Lagrangian

$$
\mathcal{L}(a, \dot{a}, \phi, \dot{\phi})=\frac{1}{N}\left(-3 a \dot{a}^{2}+\frac{\lambda}{2} a^{3} \dot{\phi}^{2}\right)-N\left(a^{3} \Lambda+\rho_{m 0} e^{\frac{\phi}{2}} a^{3-3 \gamma}\right) .
$$

\section{Integrability Property and Analytic Solution}

Since the field equations admit a point-like Lagrangian various techniques inspired by analytic mechanics be applied for the study of the dynamical system. Indeed, variational symmetries and conservation laws can be determined by using Noether's theorems [37]. That approach has been widely used in various gravitational systems. New integrable 
cosmological models as also new analytic and exact solutions were found through the use of variational symmetries, see for instance [38].

We investigate for variational symmetries which have point transformations as generators and provide conservation laws linear in the velocities. Hence, for the Lagrangian function (41) and for $\rho_{m 0} \neq 0$, we find that the variational symmetry $X=\frac{2}{3} a \partial_{a}+4(\gamma-2) \partial_{\phi}$ exists for $\Lambda=0$, and the corresponding conservation law is

$$
F(a, \dot{a}, \phi, \dot{\phi})=4 a^{2} \dot{a}-4(\gamma-2) \lambda a^{3} \dot{\phi}-F_{0} .
$$

Function $F(a, \dot{a}, \phi, \dot{\phi}), \frac{d F}{d t}=0$, is the second-conservation law for the dynamical system, which means that the field equations form an integrable dynamical system.

In order to reduce the field equations and determine exact solutions, we apply the Hamilton-Jacobi approach. We define the momentum $p_{a}=-6 a \dot{a}$, and $p_{\phi}=\lambda a^{3} \dot{\phi}$., thus the Hamiltonian function $\mathcal{H}\left(a, \phi, p_{a}, p_{\phi}\right)=0$, reads

$$
-\frac{p_{a}^{2}}{6 a}+\frac{p_{\phi}^{2}}{\lambda a^{3}}+2\left(a^{3} \Lambda+\rho_{m 0} e^{\frac{\phi}{2}} a^{3-3 \gamma}\right)=0
$$

while the Hamilton-Jacobi equation is written in the following form

$$
-\frac{1}{6 a}\left(\frac{\partial}{\partial a} S(a, \phi)\right)^{2}+\frac{1}{\lambda a^{3}}\left(\frac{\partial}{\partial \phi} S(a, \phi)\right)^{2}+2 \rho_{m 0} e^{\frac{\phi}{2}} a^{3-3 \gamma}=0,
$$

where now $p_{a}=\frac{\partial S}{\partial a}$ and $p_{\phi}=\frac{\partial S}{\partial \phi}$. $S(a, \phi)$

Moreover, the conservation law (44) provides the constraint equation for the Action

$$
\frac{2 a}{3}\left(\frac{\partial}{\partial a} S(a, \phi)\right)+4(\gamma-2) \frac{\partial}{\partial \phi}(S(a, \phi))-F_{0}=0 .
$$

We define the new variable $\phi=6(\gamma-2) \ln a+\Phi$, such that the constraint equation becomes

$$
\frac{2}{3} a \frac{\partial}{\partial a}(S(a, \Phi))-F_{0}=0
$$

This new set of variables $\{a, \Phi\}$ are the normal coordinates for the dynamical system.

Consequently, in the normal variables the analytic expression for the Action as provided by the Hamilton-Jacobi equation is

$$
\begin{aligned}
& S(a, \Phi)=\frac{3}{2} F_{0} \ln a+\int \frac{\sqrt{2 \lambda} \sqrt{16 \rho_{m 0} e^{\frac{\Phi}{2}}\left(6 \lambda(\gamma-2)^{2}-1\right)+3 F_{0}}+6 \lambda(\gamma-2) F_{0}}{4\left(6 \lambda(\gamma-2)^{2}-1\right)} d \Phi \\
& \text { for }\left(6 \lambda(\gamma-2)^{2}-1\right) \neq 0 \text {, or } \\
& \qquad S(a, \Phi)=\frac{3}{2} F_{0} \ln a+\frac{3 F_{0}^{2} \Phi-32 \rho_{0} e^{\frac{\Phi}{2}}}{24 F_{0}(\gamma-2)},
\end{aligned}
$$

when $\left(6 \lambda(\gamma-2)^{2}-1\right)=0$.

However, in the new coordinates the momentum are defined as

$$
\begin{gathered}
p_{a}=-6 a\left(\left(6 \lambda(\gamma-2)^{2}-1\right) \dot{a}+(\gamma-2) \lambda a \dot{\Phi}\right), \\
p_{\Phi}=-\lambda a(6(\gamma-2) \dot{a}+a \dot{\Phi})
\end{gathered}
$$


which give the following expressions for the scale factor and the scalar field

$$
\begin{gathered}
6 a^{2} \dot{a}=a p_{a}-6(\gamma-2) p_{\Phi} \\
\lambda a^{3} \dot{\Phi}=-p_{\Phi}-\lambda(\gamma-2)\left(A p_{A}+6(\gamma-2) p_{\Phi}\right) .
\end{gathered}
$$

Hence, by using the Action (49) and expressions (53), (54), the cosmological field equations can be written into an equivalent system. We summarize the results in the following proposition.

Proposition 1. The field equations in WIG for a FLRW background space with zero spatial curvature and an ideal gas form a Liouville integrable system when there is no cosmological constant term. The analytic solution for the Hamilton-Jacobi equation provides the Action (49), while the field equations can be written into an equivalent set of two first-order ordinary differential Equations (53) and (54).

Assume now the simple case for which $\gamma=1$ and $F_{0}=0$. Moreover, we define the new variable $T=T(t)$, such that $d T=\frac{\sqrt{(6 \lambda-1)}}{A^{3}} d t$ and $\lambda \neq \frac{1}{6}$.

Thus, the field equations are

$$
\begin{gathered}
\frac{\dot{a}}{a}-\sqrt{2 \lambda \rho_{m 0}} e^{\frac{\Phi}{4}}=0, \\
\dot{\Phi}-\sqrt{\frac{2}{\lambda} \rho_{m 0}(6 \lambda+1)} e^{\frac{\Phi}{4}}=0,
\end{gathered}
$$

with exact solution

$$
a(t)=a_{0} t^{\frac{4 \lambda}{1+6 \lambda}}, \Phi(t)=-2 \ln \left(\frac{(6 \lambda+1) \rho_{m 0}}{8 \lambda} t^{2}\right) .
$$

For this exact solution the background space is

$$
d s^{2}=-\frac{(6 \lambda-1)}{a_{0}^{6}} t^{-\frac{24 \lambda}{1+6 \lambda}} d T^{2}+a_{0}^{2} \frac{8 \lambda}{1+6 \lambda}\left(d x^{2}+d y^{2}+d z^{2}\right) .
$$

The later solution describes a universe dominated by a perfect fluid source with constant equation of state parameter. This specific solution is described by the stationary points $A_{2}$, thus. the results are in agreement with the asymptotic analysis for the dynamics.

\section{Conclusions}

In this work we considered WIG to describe the cosmological evolution for the physical parameters in FLRW spacetime with zero spatial curvature. The gravitational field equations in WIG are of second-order and Einstein's theory, with the presence of the of a scalar field, is recovered. Scalar field plays the role for conformal factor which relates the connection of Weyl theory with the Levi-Civita connection of Riemannian geometry. However, the field equations differ when matter is introduced in the gravitational model. Indeed, in WIG the matter source interacts with the scalar field. The interaction term is introduced naturally from the geometric character of the theory.

In our study we considered the matter source to be described by that of an ideal gas, that is $p_{m}=(\gamma-1) \rho_{m}$, or by the Chaplygin gas $p_{m}=-\frac{A_{0}}{\rho_{m}^{\alpha}}$. We defined new dimensionless variables based on the Hubble-normalization in order to write the field equations as a system of first-order algebraic differential system. In each model, we determined the stationary points for the latter system and we determined their dynamical properties as also the physical properties of the asymptotic solutions. In our analysis we also considered a non-zero cosmological constant. 
For the ideal gas, we found that there exists an attractor with an asymptotic solution of an ideal gas, but with a different parameter for the equation of state. For instance, we can consider the matter source to be that of radiation while the attractor to describe an accelerated universe. In the presence of the cosmological constant, we find two asymptotic solutions which can describe the past acceleration phase of the universe known as inflation, as also the late time acceleration. The future attractor describes the de Sitter universe. When the matter component is that of a Chaplygin gas the stationary points as also the cosmological evolution are similar with the previous case.

Moreover, for the ideal gas case, we solved the inverse problem and determined a Lagrangian function, and a minisuperspace description, which generates the cosmological equations under a variation. We applied Noether's theorems for point transformations in order to construct a non-trivial conservation law when the cosmological constant term is zero. Hence, the cosmological field equations form a Liouville integrable dynamical system. The closed-form expression for the Hamilton-Jacobi equation derived. Finally, for specific values for the free parameters, we were able to construct an exact solution which is in agreement with the asymptotic analysis.

In a subsequent analysis we plan to investigate further the field equations as a Hamilton system and understand how a non-zero cosmological constant affects the integrability property of the field equations.

Funding: This research received no external funding.

Institutional Review Board Statement: Not applicable.

Informed Consent Statement: Not applicable.

Data Availability Statement: Not applicable.

Conflicts of Interest: The author declare no conflict of interest.

\section{References}

1. Aghanim, N.; Akrami, Y.; Ashdown, M.; Aumont, J.; Baccigalupi, C.; Ballardini, M.; Banday, A.J.; Barreiro, R.B.; Bartolo, N.; Basak, S.; et al. Planck 2018 results. VI. Cosmological parameters. Astron. Astrophys. 2020, 641, A6.

2. Perivolaropoulos, L.; Skara, F. Challenges for $\Lambda$ CDM: An update. arXiv 2021, arXiv:2105.05208.

3. Ratra, B.; Peebles, P.J.E. Cosmological consequences of a rolling homogeneous scalar field. Phys. Rev. D 1988, 37, 3406. [CrossRef]

4. Armendariz-Picon, C.; Mukhanov, V.F; Steinhardt, P.J. Essentials of k-essence.Phys. Rev. D 2001, 63, 103510. [CrossRef]

5. Faraoni, V. Cosmology in Scalar-Tensor Gravity; Kluwer Academic Publishers: Dordrecht, The Netherlands, 2004.

6. Bento, M.C.; Bertolami, O.; Sen, A.A. Generalized Chaplygin gas and CMBR constraints. Phys. Rev. D 2003, 67, 063003. [CrossRef]

7. Kamenshchik, A.; Moschella, U.; Pasquier, V. An alternative to quintessence. Phys. Lett. B 2001, 511, 265-268. [CrossRef]

8. Basilakos, S.; Mavromatos, N.E.; Solà Peracaula, J. Gravitational and chiral anomalies in the running vacuum universe and matter-antimatter asymmetry. Phys. Rev. D 2020, 101, 045001. [CrossRef]

9. Clifton, T.; Ferreira, P.G.; Padilla, A.; Skordis, C. Modified gravity and cosmology. Phys. Rept. 2012, 513, 1-189. [CrossRef]

10. Nojiri, S.I.; Odintsov, S.D. Introduction to modified gravity and gravitational alternative for dark energy. IJGMMP 2007, 4, 115-145. [CrossRef]

11. Valentino, E.D.; Mena, O.; Pan, S.; Visinelli, L.; Yang, W.; Melchiorri, A.; Mota, D.F.; Riess, A.G.; Silk, J. In the Realm of the Hubble tension a Review of Solutions. Class. Quantum. Grav. 2021, 38, 153001. [CrossRef]

12. Billyard, A.P.; Coley, A.A. Interactions in scalar field cosmology. Phys Rev D 2000, 61, 083503. [CrossRef]

13. Pan, S.; Sharov, G.S.; Yang, W. Field theoretic interpretations of interacting dark energy scenarios and recent observations. Phys. Rev. D 2020, 101, 103533. [CrossRef]

14. Yang, W.; Mukherjee, A.; Di Valentino, E.; Pan, S. Interacting dark energy with time varying equation of state and the H0 tension. Phys. Rev. D 2018, 98, 123527. [CrossRef]

15. Pan, S.; Sharov, G.S. A model with interaction of dark components and recent observational data. MNRAS 2017, 472, 4736-4749. [CrossRef]

16. Di Valentino, E.; Melchiorri, A.; Mena, O.; Vagnozzi, S. Interacting dark energy in the early 2020s: A promising solution to the H0 and cosmic shear tensions. Phys. Dark Univ. 2020, 30, 100666. [CrossRef]

17. Yu Vernov, S.; Pozdeeva, E. De Sitter Solutions in Einstein-Gauss-Bonnet Gravity. Universe 2021, 7, 149. [CrossRef]

18. Yu Kamenshchik, A.; Pozdeeva, E.O.; Venturi, G.; Yu Vernov, S. Integrable cosmological models in the Einstein and in the Jordan frames and Bianchi-I cosmology. Phys. Part. Nucl. 2018, 49, 1-4. [CrossRef] 
19. Dimakis, N.; Paliathanasis, A.; Terzis, P.A.; Christodoulakis, T. Cosmological solutions in multiscalar field theory. EPJC 2019, 79, 618. [CrossRef]

20. Paliathanasis, A. De Sitter and scaling solutions in a higher-order modified teleparallel theory. JCAP 2017, 8, 027. [CrossRef]

21. Copeland, E.J.; Liddle, A.R.; Wands, D. Exponential potentials and cosmological scaling solution. Phys. Rev. D 1998, 57, 4686. [CrossRef]

22. Coley, A.; Leon, G. Static Spherically Symmetric Einstein-aether models I: Perfect fluids with a linear equation of state and scalar fields with an exponential self-interacting potential. Gen. Rel. Grav. 2019, 51, 115. [CrossRef]

23. Amendola, L.; Polarski, D.; Tsujikawa, S. Are f(R) dark energy models cosmologically viable? Phys. Rev. Lett. 2007, 98, 131302. [CrossRef] [PubMed]

24. Amendola, L.; Gannouji, R.; Polarski, D.; Tsujikawa, S. Conditions for the cosmological viability of f(R) dark energy models. Phys. Rev. D 2007, 75, 083504. [CrossRef]

25. Christodoulidis, P.; Roest, D.; Sfakianakis, E.I. Scaling attractors in multi-field inflation. JCAP 2019, 12, 059. [CrossRef]

26. Fadragas, C.R.; Cardenas, R.; Rodriguez-Ricard, M.; Rivero-Acosta, A.; Linares-Rodriguez, A. Detailed qualitative dynamical analysis of a cosmological Higgs field. Gen. Rel. Gravit. 2019, 51, 109. [CrossRef]

27. Gonzalez, T.; Leon, G.; Quiros, I. Dynamics of quintessence models of dark energy with exponential coupling to dark matter. Class. Quantum Grav. 2006, 23, 32165. [CrossRef]

28. Kerachia, M.; Acquaviva, G.; Lukes-Gerakopoulos, G. Dynamics of classes of barotropic fluids in spatially curved FRW spacetimes. Phys. Rev. D 2020, 101, 043535. [CrossRef]

29. Aguilar, J.E.M.; Romero, C. Inducing the cosmological constant from five-dimensional Weyl space. Found. Phys. 2009, 39, 1205-1216. [CrossRef]

30. Liu, Y.-X.; Yang, K.; Zhong, Y. de Sitter Thick Brane Solution in Weyl Geometry. JHEP 2010, 10, 069. [CrossRef]

31. Lobo, I.P.; Barreto, A.B.; Romero, C. Space-time singularities in Weyl manifolds. EPJC 2015, 75, 448. [CrossRef]

32. Paliathanasis, A.; Leon, G. Integrability and cosmological solutions in Einstein-æther-Weyl theory. EPJC 2021, 81, 255. [CrossRef]

33. Pucheu, M.L.; Alves, F.A.P., Jr.; Barreto, A.B.; Romero, C. Cosmological models in Weyl geometrical scalar-tensor theory. Phys. Rev. D 2016, 94, 064010. [CrossRef]

34. Miritzis, J. Isotropic cosmologies in Weyl geometry. Class. Quantum Grav. 2004, 21, 3043. [CrossRef]

35. Salim, J.M.; Sautú, S.L. Gravitational collapse in Weyl integrable space-times. Class. Quantum Grav. 1999, 16, 3281. [CrossRef]

36. Salim, J.M.; Sautú, S.L. Gravitational theory in Weyl integrable spacetime. Class. Quantum Grav. 1996, 13, 353. [CrossRef]

37. Halder, A.; Paliathanasis, A.; Leach, P.G.L. Noether's Theorem and Symmetry. Symmetry 2018, 10, 744. [CrossRef]

38. Tsampalis, M.; Paliathanasis, A. Symmetries of Differential Equations in Cosmology. Symmetry 2018, 10, 233. [CrossRef] 